\title{
Futuna, catalyseur de la symbiose des deux artistes : Aloi Pilioko et Nicolaï Michoutouchkine
}

Christian Coiffier

\section{(2) OpenEdition \\ 12 Journals}

Édition électronique

URL : http://journals.openedition.org/jso/619

DOI : $10.4000 /$ jso. 619

ISSN : $1760-7256$

Éditeur

Société des océanistes

Édition imprimée

Date de publication : 1 décembre 2006

Pagination : 173-186

ISSN : 0300-953x

\section{Référence électronique}

Christian Coiffier, «Futuna, catalyseur de la symbiose des deux artistes : Aloi Pilioko et Nicolai

Michoutouchkine », Journal de la Société des Océanistes [En ligne], 122-123 | Année 2006, mis en ligne le 01 décembre 2009, consulté le 21 avril 2019. URL : http://journals.openedition.org/jso/619 ; DOI : $10.4000 /$ jso.619 


\title{
Futuna, catalyseur de la symbiose des deux artistes : Aloi Pilioko et Nicolaï Michoutouchkine
}

par

\author{
Christian COIFFIER*
}

\section{RÉSUMÉ}

Les arts non occidentaux sont à la mode avec l'ouverture du musée du quai Branly à Paris. Mais sait-on que depuis plus de quarante-cinq ans deux artistes, Aloi Pilioko et Nicolä Michoutouchkine ont consacré toute leur énergie pour promouvoir l'art du Pacifique, sans parti pris entre les arts traditionnels et les arts contemporains! Aloi Pilioko est originaire de l'île de 'Uvea (Wallis) ; Nicolaï Michoutouchkine, français d'origine russe, est tombé sous le charme de l'île de Futuna. Nous verrons ainsi comment les îles Wallis-et-Futuna représentent le lieu de gestation de l'intérêt de ces deux artistes pour le Pacifique.

Mots-CLÉs : Wallis-et-Futuna, art du Pacifique, artiste, collectionneur, muséographie.

L'année 2006 sera marquée à Paris par l'ouverture d'un nouveau musée d'art. Ce projet présidentiel, initié par le marchand-collectionneur Jacques Kerchache, aura connu une gestation de dix années pour arriver à son terme. La fonction annoncée de cette institution qui portera le nom de musée du quai Branly sera la promotion des arts extra-européens, dont font partie les arts du Pacifique. Depuis une décennie, ces arts exotiques sont à la mode. Ce n'était guère le cas dans les années 1960 lorsque deux pionniers, les peintres Aloi Pilioko et Nicolaï

\begin{abstract}
Non Western art has become fashionable with the opening of the Quai Branly Museum in Paris. Nevertheless, in spite of this change in European attitudes, for more than forty-five years two artists, Aloi Pilioko and Nicolas Michoutouchkine, have devoted all their energy in order to promote Pacific art without favouring either traditional or contemporary art. We shall show how Wallis and Futuna islands have caused these two artists -Pilioko born on the 'Uvea island (Wallis) and Michoutouchkine born in France of Russian decent - to succumb to the charm of Futuna.
\end{abstract}

Keywords: Wallis and Futuna Islands, Pacific art, artist, collector of curios, museography.

Michoutouchkine commencèrent à exposer les premières pièces de leur collection d'objets océaniens en les associant à leur propre production artistique. Le mélange de genres n'était pas dans l'air du temps à cette époque, pourtant les deux artistes sont considérés aujourd'hui comme les précurseurs de l'art contemporain au Vanuatu (Regenvanu, 1996 : 322), comme le reconnaît le sculpteur Emmanuel Watt dans un entretien publié il y a quelques années dans le journal Mwà véé (2002: 49 et 56). Depuis plus de quarante ans, Pilioko et Michoutouchkine n'ont

* Maître de conférences au Muséum national d'histoire naturelle, chargé de mission au musée du quai Branly, coiffier@mnhn.fr 
cessé de promouvoir, avec une rare opiniâtreté, leur vision des arts du Pacifique à travers le monde en présentant plus d'une centaine d'expositions. Tous deux sont membres de la Société des Océanistes depuis de nombreuses années. Nous évoquerons l'œuvre et le parcours d'Aloi Pilioko qui ne peut être séparé de celui de son alter ego Nicolaï Michoutouchkine. Ces deux artistes, plus connus localement par leurs prénoms Aloi et Nicolaï, se sont forgés en effet conjointement un destin hors du commun dans lequel les îles Wallis et Futuna ont joué un rôle essentiel, voire fondamental.

\section{Les origines : 'Uvea et Futuna}

Aloi Pilioko (1980) se présente lui-même dans une plaquette bilingue (anglais/français) abondamment illustrée et intitulée Pilioko, artiste du Pacifique. Il est né à 'Uvea, en 1935, dans une famille de onze enfants. Son père, nommé Silino Maufehi était un charpentier tufuga, constructeur de maison, chef du village de 'Alele et grand officiant pour les cérémonies du kava, connu et apprécié dans toute l'île. Il était également très respecté pour s'être jadis opposé au Résident David, raison pour laquelle il avait été exilé à l'extrémité de l'île, dans le district de Mu'a. La jeunesse de Pilioko s'est donc déroulée chez sa tante à Lavegahau. Deux membres de sa famille étaient des ecclésiastiques : sa sœur aînée, missionnaire mariste à Futuna, et l'un de ses frères, prêtre de la paroisse de Sigave (Futuna) après avoir été missionnaire à Samoa sur l'invitation du Cardinal Pio Taufinuu. En 1956, Aloi s'expatrie aux Nouvelles-Hébrides (l'actuel Vanuatu) pour travailler dans une plantation d'Epi, avant d'aller, en 1958, à la recherche d'un autre emploi en Nouvelle-Calédonie. L'ouvrage Le Russe de Belfort de Marie-Claire Teissier-Landgraf, publié en 1995, avec le sous-titre 37 ans de voyages du peintre Nicolaï Michoutouchkine en Océanie vient compléter cette histoire, car la vie et l'œuvre des deux artistes Michoutouchkine et Pilioko se confondent à partir de leurs rencontres. Ce livre montre l'influence primordiale et salutaire dont ils bénéficièrent lors de leur séjour dans les deux îles de 'Uvea et de Futuna. Nicolaï l'écrit lui-même dans une présentation de sa collection :

«Sans doute est-ce par les liens créés avec Futuna, les Futuniens et leur art, durant deux ans, que je pris conscience moi-même d'une responsabilité ; sauvegarder leur patrimoine à la dérive, voué à la disparition ou à la revente au profit de quelques chasseurs d'Art primitif. » (Egami, $1980: 118)$
Nicolaï Michoutouchkine, fils d'émigré russe, est né à Belfort en 1929 où il a fait ses études secondaires. Sa jeunesse baigne dans le mysticisme familial des vieilles traditions russes. Le conservateur du musée de la ville, $\mathrm{M}$. Delarbre et le peintre Bourland, ami de Matisse, remarquent ses dons pour le dessin. En 1949, après un voyage en auto-stop à Rome, il a l'intention de devenir prêtre orthodoxe, mais il abandonne ce projet pour venir à Paris suivre des études dans une école de Sciences commerciales. Il s'inscrit parallèlement dans l'atelier de peinture de la Grande Chaumière à Montparnasse afin de perfectionner son talent pour le dessin. Mais Michoutouchkine est attiré par l'Orient et les grands horizons. Une fois ses études terminées, il entreprend à partir d'août 1953 un long voyage qui va le mener à travers les pays les plus divers jusqu'en Océanie. Au cours de ces quatre années d'errance, son entregent lui permet de nouer de bonnes relations avec les services culturels français et les ambassades des pays qu'il traverse. Ces contacts lui donnent l'occasion de rencontrer de nombreuses personnalités politiques; le président du Liban Camille Chamoun, le roi Hussein de Jordanie, le président égyptien Néguib, le Shah d'Iran, Madame Indira Gàndhi, le Premier ministre de Ceylan le Dr. Radarrishnan, le roi du Népal, le dalaï-lama et bien d'autres personnalités encore... Lors d'une de ses étapes à Beyrouth son talent sera remarqué par l'historien de l'art Gaetan Picon. Nicolaï organise dans divers pays visités des expositions de ses œuvres, réalisées sur place, particulièrement en Inde et en Birmanie où il se passionne pour la spiritualité bouddhique. Il développe alors son goût pour l'orientalisme et affirme sa personnalité originale au contact de ses diverses rencontres comme Élisabeth Brunner, disciple du poète hindou Rabindranath Tagore (Teissier-Landgraf, $1995: 83$ ). L'Inde sera le révélateur de sa recherche personnelle qui portera ses fruits un peu plus tard à Futuna (Gaudron, 1983 : 1).

Mais, en 1957, il est convoqué par l'administration française pour faire son service militaire car son sursis est arrivé à expiration. Il quitte alors précipitamment Sydney où il vient d'exposer ses dessins. Il rejoint le plus vite possible l'établissement français le plus proche, en l'occurrence la Nouvelle-Calédonie, et il est incorporé en novembre à Nouméa. Il a cependant le temps d'exposer ses œuvres, inspirées par ses derniers séjours en pays bouddhiques, à la Société havraise calédonienne de Nouméa. Après une affectation auprès du commandant des Forces armées de Nouvelle-Calédonie, il devient secrétaire particulier du gouverneur Gri- 
mald. Ce poste lui donne l'occasion de voyager à travers tout le territoire et de découvrir l'art kanak, ce qui représente sa première initiation à l'art océanien. Le jeune homme fait vite connaissance avec l'intelligentsia et le milieu artistique local. Il découvre l'art wallisien par l'intermédiaire d'un immense tapa qui décore la maison de son colonel (Teissier-Landgraf, 1995 : 14). Après sa démobilisation, l'Office du tourisme de Nouméa lui demande de constituer une collection d'art kanak contemporain. Sa vocation de collectionneur est née, ce qui va l'inciter à ouvrir très rapidement une des premières galeries d'art $\mathrm{du}$ Pacifique francophone sur les quais de Nouméa à côté du café Grazziani. Il y expose conjointement des objets d'artisanat local avec des œuvres d'artistes comme Frank Fay, Tatin d'Avesnières, Marcel Nicolas ou Jemmanick... C'est cette même année qu'un jeune wallisien, nommé Aloi, fait sa connaissance dans la galerie d'art. Nicolaï découvre rapidement les talents d'artiste du jeune homme et l'encourage tout en respectant sa personnalité. L'apprentissage devient réciproque et les deux artistes vont continuer par la suite à développer leur propre sensibilité au contact l'un de l'autre.

Suite à une annonce dans le journal La France Australe, Nicolaï est recruté comme directeur d'un magasin appartenant à une société chargée de la commercialisation du coprah à Futuna (Mwà véé, 2004: 59). Il décide donc de partir s'établir dans cette île où il arrive, en décembre 1959, sur le Maria des Mar. À cette époque, Futuna n'est desservie que tous les trois mois par un bateau faisant la navette entre Nouméa et Sigave. Il n'y a pratiquement pas de routes à l'intérieur de l'île et le cabotage est rendu difficile et dangereux par les récifs coralliens. Cette île représente donc pour le jeune homme l'île merveilleuse de ses rêves de jeunesse, il va pouvoir effectuer un voyage autour de lui-même pour faire le point après ces années d'errance en Orient et en Asie. Il s'adapte très vite à son emploi et à sa nouvelle vie, il s'extasie devant la beauté de la population, il admire l'audace avec laquelle les femmes choisissent les couleurs de leur pagne et le port altier des hommes au corps musclé ceint de leur lava.

« La peinture de Nicolaï commence à prendre des teintes plus chaudes, comme éclairées par le soleil. » (Teissier-Landgraf, $1995: 24$ )

La tonalité générale des œuvres du peintre était en effet beaucoup plus terne avant son arrivée à Futuna.

Trois mois plus tard, Aloi Pilioko vient le rejoindre. Les deux années passées à Sigave sont très profitables pour la formation générale du nouvel artiste et vont influencer sa carrière future. C'est là que ce dernier va découvrir l'art de la broderie auprès de Louise Varin, une amie de Nicolaï. Cette découverte lui donne l'idée de concevoir une nouvelle technique qu'il va perfectionner et qui sera par la suite la source de son succès. Il en profite pour parfaire ses connaissances en ce qui concerne la médecine traditionnelle locale et perfectionne sa pratique de la langue française. Il rencontre les dignitaires politiques comme Veu, le Tamolevai de Sigave, le Tui Agaifo d'Alo et Tomasi Kulimoetoke, le Lavelua de Wallis. Toutes ces activités et ses rencontres vont alors stimuler son esprit créatif et donner naissance à des œuvres très diverses. Au cours de leurs déplacements dans l'île, Aloi et Nicolaï, tous deux passionnés par les beaux objets, achètent de nombreux produits de fabrication locale (cf. les catalogues : Egami, 1980, Bromley, 1989); lances tao, bâtons de chef tokotoko, hameçons divers $p \bar{a}$, haches de pierre, colliers kasoa, pendentifs lei et pule, plats kumete et tāno'a, mortier pour écraser le kava makasinu, palettes de dance, kailao et paki, éventails ili, ceintures lafi, jupes tepi, nattes tressées, matrices à tapa kupeti, et surtout des tapas de toutes sortes : lafi, salatasi, salatolu et lausiapo, travail féminin par excellence pouvant atteindre plusieurs mètres (Gaillot, 1963: 213, TeissierLandgraf, $1995: 28-29)$. Nicolaï est particulièrement sensible au fait que les Futuniens arrivent à fabriquer toutes sortes d'objets très esthétiques avec un choix relativement restreint de matières premières (Teissier-Landgraf, $1995: 29$ ). Il rêve de conserver sur place tous les objets qu'il a collectés dans un petit musée qu'il souhaiterait faire construire sur un terrain situé à Sigave car il se rend compte de la désaffection progressive des Futuniens pour leur art coutumier et déplore que ce fait soit encouragé par l'Église.

«Son enthousiasme et sa sincérité l'empêchent de comprendre qu'il va à l'encontre de certaines opinions partagées par beaucoup de Français métropolitains de l'époque : il n'est pas de bon ton de susciter un réveil de la culture locale. » (Teissier-Landgraf, 1995 : 29)

Son projet n'est soutenu ni par l'administration locale ni par la métropole, ce qui radicalise un peu plus son opinion sur les effets de la présence européenne en Océanie.

« Il perçoit de mieux en mieux l'effet pervers de déculturation entrepris dans cette région depuis plus de cent ans par le christianisme qui impose, entre autre, sa civilisation de béton et de tôles ondulées. » (Teissier-Landgraf, $1995: 30$ ) 


\section{Installation à Port-Vila}

Déçus, Pilioko et Michoutouchkine quittent Futuna, en août 1961, et emportent alors, à regret, leur collection d'objets de Futuna ainsi que plus de cent trente œuvres comprenant des huiles réalisées sur des nattes en vannerie et d'autres sur papier, avec également des aquarelles. Ce séjour a prouvé, pour le moins, à Nicolaï qu'il pouvait vivre aisément à la manière océanienne, sans téléphone ni électricité, en dormant à même une natte. Les deux artistes partent alors pour aller organiser une exposition sur Futuna dans le tout nouveau Centre Culturel de PortVila aux Nouvelles-Hébrides (Vanuatu), sous le patronage de la Résidence de France. Ils sont encouragés dans cette entreprise par maître Degranges $^{1}$, ancien avocat et homme cultivé, qui apprécie leur peinture et leur démarche de collectionneurs (Mwà véé, 2004 : 55). Michoutouchkine organise au mois de décembre suivant de la même année, avec une série d'œuvres de Pilioko, une autre exposition sur Futuna à l'Institut français de l'Océanie à Nouméa (le futur ORSTOMIRD). Dans le premier numéro spécial du JSO de décembre 1963 consacré à Wallis-et-Futuna (pp. 211-214), Marcel Gaillot présente l'œuvre d'un jeune artiste français, Nicolaï Michoutouchkine, qui vient de vivre près de deux années à Futuna. Il raconte comment ce dernier y a vécu de façon très spartiate pour un Européen et comment, au bout de quelques semaines, sa bonne connaissance des rudiments de la langue futunienne lui attira la sympathie de la population. L'île de Futuna se révèle être très favorable à l'inspiration de l'artiste, il croque des paysages, des scènes de la vie quotidienne et il réalise de nombreux portraits. Marcel Gaillot conclut ainsi laconiquement son article :

«Son séjour à Futuna et l'œuvre qu'il y a réalisée auront eu le grand mérite de faire connaître à quelques milliers de personnes, cette île isolée, que nous n'avons plus le droit d'ignorer. »

Les années qui suivent le retour de Futuna sont riches en voyages à travers tout le Pacifique et en visite de nombreuses îles : les NouvellesHébrides dont, Ambrym, Espiritu Santo, Malakula, Vao, Atchin, Wala-Rano, la Polynésie française dont Tahiti, Bora-Bora, Huahine, Maupiti, Raiatea, Anaa, les îles Salomon dont
Guadalcanal, Santa Ana, Santa Catalina, Santa Cruz, Malaita, Makira, Vanikoro, Utupua et Tikopia, la Nouvelle-Guinée avec une descente du fleuve Sépik et une visite des Highlands, l'Australie dont Brisbane, Sydney, Canberra et finalement Adélaïde où Pilioko est invité à participer au premier Festival des Arts du Pacifique. Durant ces voyages, il a l'occasion de rencontrer d'autres artistes polynésiens comme lui ; Mara des Australes, Rui Juventin, des peintres européens installés à Tahiti comme Fay, Masson, Grès, Gouwe et Vahine Terupe (Pilioko, 1980 : 14) et évidemment des personnalités locales comme les grands chefs de Tikopia, Teariki Taumako, Kafika, Fangarere, la reine Salote de Tonga, les grands chefs Kali et Viriambat de Malakula aux Nouvelles-Hébrides, le père Marion Ganey à Fidji, monseigneur Darmencier à Wallis et Futuna et bien d'autres encore (Teissier-Landgraf, $1995: 85$ ).

En mai 1964, c'est le retour à Port-Vila et le début de la construction de maisons et d'ateliers sur un terrain situé sur la route du village de Pango au bord du lagon au lieu-dit " Esnaar», acheté en 1963 à Maître Desgranges et payé pour moitié en tableaux (Mwà véé, 2004 : 55). Cet endroit va devenir le lieu de résidence privilégié des deux artistes jusqu'à aujourd'hui. Les Nouvelles-Hébrides présentent, à cette époque, de nombreux avantages aux yeux des deux artistes. Ce pays n'est pas encore trop touché par l'occidentalisation et les gens y sont très hospitaliers ; c'est un lieu de rencontre des espaces polynésien et mélanésien, où l'on parle deux langues étrangères, le français et l'anglais, en plus du bislama et des nombreuses langues locales (Egami, 1980 : 118-119). Ce n'est pas ce point d'ancrage qui va réfréner leur errance transPacifique, bien au contraire, ils repartent pour présenter leurs collections d'objets et d'œuvres personnelles. C'est de nouveau la NouvelleCalédonie qui les accueille, puis Tonga, et Suva dans l'archipel de Fidji. Tonga retient particulièrement l'attention de Pilioko car il espère $\mathrm{y}$ retrouver des communautés wallisiennes. Il est particulièrement ému lorsqu'il entend dans les villages résonner le bruit caractéristique des battoirs des femmes qui assouplissent les fibres végétales destinées à la fabrication des tapas. À Rotuma, il se trouve à manquer de peinture, cela lui donne l'idée d'utiliser des fils de laine de

1. Maître Desgranges, personnalité éminente de Port-Vila, connaissait très bien le milieu artistique parisien et, entre autres, Georges-Henri Rivière. On lui doit la donation pour le musée de l'Homme de la célèbre sculpture du Vanuatu, Trrou Körrou, plus connue sous le nom de l'Homme bleu, en provenance de l'île de Malo. Cette sculpture de trois mètres de hauteur fut remise aux membres de l'expédition de la Korrigane (1934-1936) qui se chargèrent de la faire convoyer en France. Elle appartient maintenant aux collections du musée du quai Branly et se trouve exposée dans une salle du palais des Sessions du musée du Louvre (Coiffier, 2000 : 274-278) où l'harmonie de ses formes fait l'admiration des visiteurs. 
couleur pour tracer sur de la toile de jute les formes que son inspiration lui dicte, à l'aide d'une aiguille à coudre les sacs de coprah. Il reconnaît lui-même avoir été influencé tant par le travail de broderie européen que par celui des femmes rotumanes qui décorent leurs nattes avec des brins de laine colorés (Pilioko, 1980 : 24-26).

En 1965, Pilioko ressent l'envie de retourner dans son île. L'occasion lui en est donnée lorsque l'Administrateur supérieur des îles Wallis-etFutuna, M. Duc Dufayard, fait organiser en son honneur une grande exposition à Mata 'Utu. Les objets de la collection sont exposés à la cathédrale et à l'Assemblée territoriale, alors que les œuvres d'Aloi Pilioko sont présentées au Palais royal. Michoutouchkine aura donc réussi le tour de force de faire apprécier des objets usuels comme œuvres d'art par leurs propres utilisateurs, les Wallisiens. La presque totalité de la population de l'île vient admirer les collections et fêter son héros local. Si cette exposition renforce le prestige de l'artiste, ce dernier ne se laisse pas troubler par cette réussite et il décide de continuer sa vie comme auparavant. Il ne veut pas se laisser enfermer dans un communautarisme étriqué car, tout en étant fier d'être wallisien, il se revendique « homme du Pacifique » qui a besoin de contacts avec le monde extérieur pour créer et exposer (Pilioko, 1980: 27). Après un rapide séjour à Futuna, les deux artistes repartent pour visiter Tahiti, les Marquises, les Australes et Samoa. Le point final de cette série de voyages sera des expositions presque simultanées à Santo, Port-Vila, Papeete et Nouméa pour les Jeux du Pacifique.

\section{Début d'une carrière internationale.}

Le succès de Pilioko ne fait que croître dans tout le Pacifique. Il a trouvé un style :

«des personnages longilignes et ondulants avec d'immenses yeux en amande, des animaux familiers dont des coqs rutilants et des poissons multicolores, des fleurs piquées ici et là, le tout exprimé avec des couleurs vives et fraîches. » (Teissier-Landgraf, 1995 : 29)

En février 1967, Nicolaï et Aloi sont invités à Paris pour présenter une exposition parrainée par la compagnie aérienne UTA dans ses salons de la place de la Madeleine. Les expositions se succèdent alors à un rythme soutenu; au musée d'Art moderne du palais de Tokyo, à l'abbaye de Royaumont et à Belfort. Pour le premier, c'est un retour dans la capitale après quatorze ans d'absence, avec deux tonnes d'objets, et pour le second, c'est la découverte d'un monde aussi étrange que celui que son ami Nicolaï avait pu percevoir à Futuna quelques années auparavant. Les expériences se trouvent alors inversées. Aloi Pilioko se transforme en ambassadeur du Pacifique dans les salons parisiens et dans diverses villes de France, il apprécie beaucoup ce rôle et s'en acquitte avec beaucoup de talent et de gentillesse. Une bourse lui est accordée par Louis Jacquinot qui lui permet de faire un stage dans les ateliers de Louise Vautrin. Son séjour parisien lui donne également l'occasion de découvrir l'Europe dont l'Espagne, l'Italie, l'Angleterre et la Suisse, où il rencontre le professeur Jean Gabus, directeur du musée d'ethnographie de Neuchâtel. L'amitié qui naîtra de cette rencontre avec les deux artistes permettra par la suite l'organisation d'une exposition. Nicolaï et Aloi retournent dans leur île en prenant le chemin des écoliers. Ce retour est jalonné d'escales à Ceylan (Sri Lankà), en Malaisie, en Thaïlande, au Vietnam et en Indonésie avec Bali. La compagnie d'aviation UTA International passe commande à Pilioko de 240 dessins pour les chambres de l'Hôtel Maeva Beach de Tahiti alors en construction. Une partie de la collection (soit environ 500 objets) y est mise en dépôt et elle s'y trouve encore actuellement (Michoutouchkine, 1970 : 60). Une exposition y est organisée peu après son ouverture en 1971, avec un catalogue, en hommage au savant russe Nikolaï MikloukhoMaklay (Michoutouchkine, $1971: 60$ ).

Pour Pilioko, c'est le début de l'utilisation de la technique des hachures, qui lui fut enseignée chez Louise Vautrin. À partir de 1970, les commandes pour la décoration de bâtiments publics se multiplient ; décoration de l'Ecole des Unions de Crédit à Fidji, maison Bergengren à Suva, Banque Indosuez de Port-Vila, décorations murales pour l'hôtel Royal Papeete à Tahiti. La Maison du Tourisme à Papeete organise une exposition de ses tableaux à l'aiguille à la fin de l'année 1971. L'année suivante est le début des honneurs pour l'artiste wallisien. Il est le premier artiste océanien à résider à l'Université du Pacifique Sud à l'occasion du troisième Festival des Arts du Pacifique. Ses panneaux et ses tapisseries seront les premières œuvres d'un artiste océanien à orner les salles et les couloirs des bâtiments universitaires. Le professeur Ron Crocombe de l'université du Pacifique Sud à Suva publie la photo d'une de ses tapisseries en première de couverture de son ouvrage The New South Pacific (1973). Les commandes ne cessent d'arriver ; il est chargé de la décoration (tapisseries et panneaux décoratifs) du nouvel hôtel d'UTA, Château Royal à Nouméa. En 1974, le jury du 


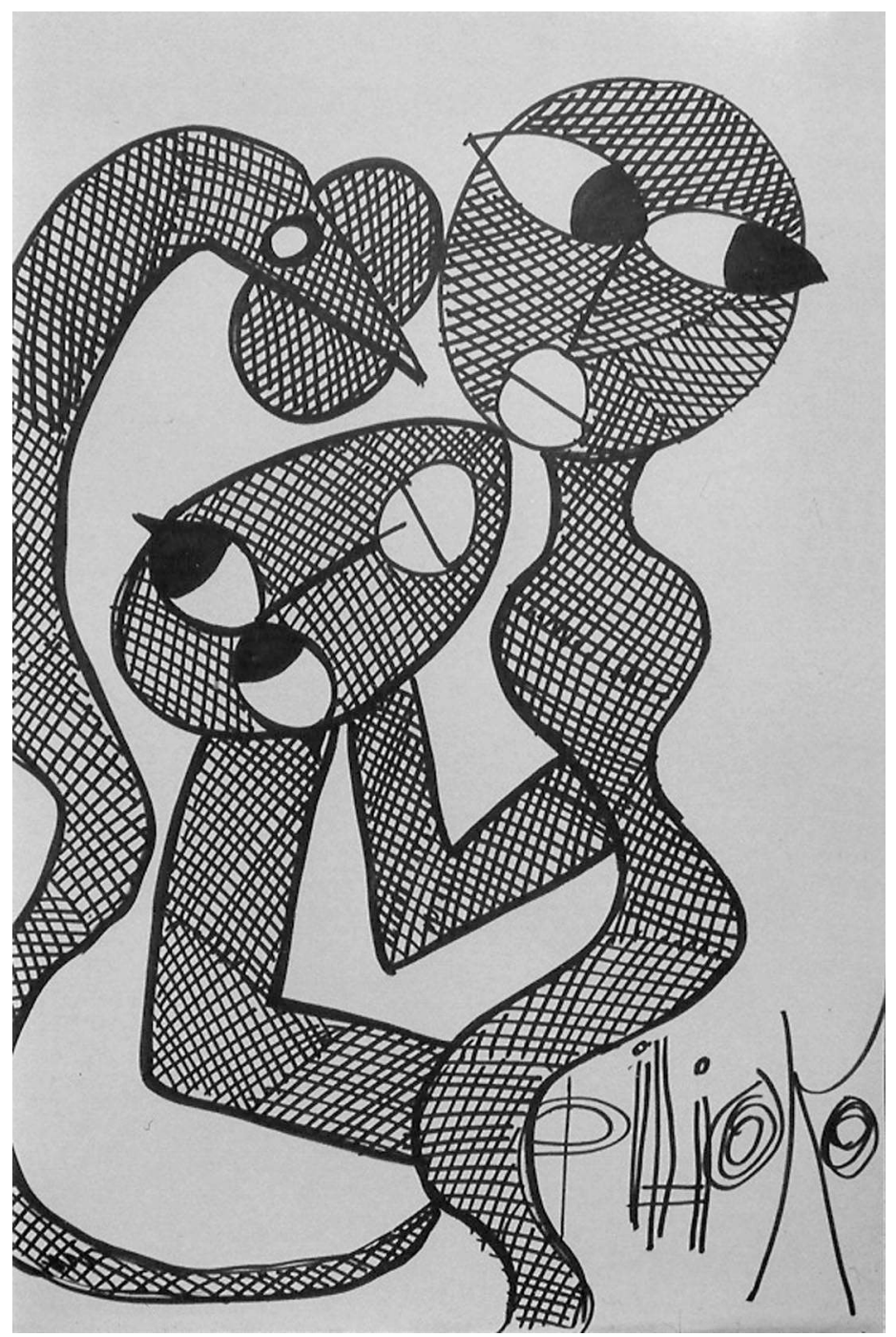

Figure 1. - Détail d'une Cuvre d'Aloi Pilioko (1977) : Coq avec personnages aux grands yeux en amande (cliché Coiffier, 1979).

concours organisé par les autorités de la ville de Port-Vila retient son projet pour la décoration de la façade du bâtiment du nouvel Hôtel des Postes. Les deux panneaux sont réalisés avec du ciment peint et décorés d'éclats de verre. L'un d'eux s'inspire de la légende du dieu Tagaloa qui aurait pêché les îles comme des poissons (Pilioko, 1980 : 38). Michoutouchkine et Pilioko font un bref séjour en Nouvelle-Zélande et exposent leurs œuvres à Auckland, puis au mois d'avril de l'année suivante, ils sont invités à Tokyo pour une exposition qui leur donne l'occasion de découvrir le Japon et la Corée. À leur retour, une rétrospective des œuvres de Pilioko est organisée au Café de Paris de Nouméa. L'artiste ressent l'envie de profiter un peu plus d'Esnaar, il se contente donc d'envoyer ses œuvres dans différents coins du monde comme au Festival des Arts d'Adélaïde et au Centre Culturel d'Ararat à Melbourne. Il est invité, en
1976, par le World Craft Council de Mexico pour représenter le Pacifique et faire en public une démonstration de son talent. À cette époque, il produit environ 15 toiles par an, réalisées à l'aiguille, qui sont vendues dès leur finition.

\section{Création d'une fondation et découverte de l'Union soviétique}

La fin des années 1970 sera marquée par deux événements particulièrement importants pour la vie professionnelle des deux peintres. En 1969, Nicolaï compte offrir à la Fondation de France les six mille pièces de sa collection et son terrain d'Esnaar en contre partie de la construction d'un musée à Port-Vila afin de remercier l'État français de l'accueil fait à ses parents immigrés de Russie. Il invite à Port-Vila le professeur Jean Gabus, directeur du Conseil International des 
Musées, et créateur de nombreux musées dans le monde, pour élaborer les plans d'aménagement de son projet. Le ministre des territoires d'Outremer confirme le financement sur le budget FIDES 1975. La Fondation de France doit se porter garante, jusqu'à la disparition des donateurs, du respect des clauses du contrat concernant cette donation. Ce dernier stipule ainsi : "Création d'un musée océanien aux Nouvelles-Hébrides, indivision de la collection, poursuite de ses buts culturels et éducatifs et de son accroissement, sa direction étant confiée à un Océanien compétent ». Le musée sera géré par un Comité de gestion et un Conseil d'anciens, inspiré des conseils coutumiers locaux, pour superviser les décisions et les activités d'animation. Henri Vallet, secrétaire financier du commissaire résident, porte le projet à bout de bras. Le professeur Georges-Henri Rivière (spécialiste des arts et traditions populaires) encourage la création de ce musée, que son initiateur espère pouvoir faire profiter de ses idées d'avant-garde: libérer l'objet de sa vitrine, utiliser des nouvelles techniques de présentation et des méthodes modernes d'animation. Une profession de foi de Michoutouchkine (1975 : 52-55) intitulée Vers un musée vivant, titre qui parodie le nom d'une célèbre revue d'art, est publiée dans la revue officielle de la Commission du Pacifique Sud. Il exprime ainsi ses idées au sujet du marché de l'art océanien :

« Il est surprenant que, malgré toutes les infrastructures de protection (musées, directions des musées, douanes, savants et doctes spécialistes), toutes ces îles (soi-disant démunies) continuent à être écumées de leurs pièces monumentales pour approvisionner le marché d'art primitif mondial, et servent à quelques collectionneurs d'investissement ou de placement [...] Cette regrettable expropriation continuera tant qu'on ne contrôlera pas mieux les exportations des pièces pour la revente à l'étranger, à moins que volontaires européens et autochtones contribuent à la création rapide de centres de sauvegarde, et ce avec discernement (Il n'est pas question, bien sûr, de priver les artisans d'un revenu vital). »

Après plusieurs années de démarches administratives, le projet échoue au dernier moment, peu de temps après la pose de la première pierre par le ministre des Territoires d'Outre-mer, lorsque monsieur Langlois, Commissaire Résident du Gouvernement français, qui l'a soutenu, est muté dans un autre poste (Egami, $1980: 119)^{2}$.
Cette année 1975 est donc marquée par la grande déception de Nicolaï qui voit tant d'efforts et d'espoirs anéantis par la volonté et les intrigues de quelques hauts fonctionnaires à Paris. Pilioko avait eu le secret espoir de voir son neveu Sioli Pilioko diriger ce nouveau musée de Port-Vila. C'est pour cela que Nicolaï avait incité et aidé celui-ci à poursuivre des études supérieures (avec des stages dans les musées) en France et en Suisse. Il sera finalement nommé chef du service des Affaires culturelles de Wallis. Nicolaï décide alors de créer sa propre association sous le vocable : «Fondation Michoutouchkine-Pilioko » dédiée à la préservation des valeurs artistiques du Pacifique. Celle-ci voit le jour en 1978 et recueille l'entier soutien et le patronage du Premier ministre Walter Lini après l'indépendance du Vanuatu (Michoutouchkine \& Pilioko Foundation, 1982 : 45). Une partie de la collection est donnée à la Little Word Foundation pour une exposition permanente dans un musée de Nagoya près d'Osaka au Japon en contrepartie de son aide pour l'organisation d'autres expositions (Egami, 1980). Le reste de la collection est exposée par la Fondation qui porte le nom des deux artistes dans l'anti-musée d'Esnaar :

« comme se plaît à le dire Nicolaï qui prône le dialogue direct, sans barrières ni vitrines, entre l'objet présenté et le visiteur. » (Mwà véé, 2004 : 52)

De nombreux enfants des écoles de Port-Vila viennent maintenant visiter chaque année ce lieu, féerique à leurs yeux, pour y admirer les objets réalisés par les anciens. En 1980, l'ancien Condominium franco-britannique des Nouvelles-Hébrides accède à l'indépendance sous le nouveau nom de Vanuatu. Une aire nouvelle commence pour cet archipel et ses habitants fiers de leur patrimoine culturel national.

Le second événement important est une grande aventure qui commence en 1977. Nicolai a perdu son père trois ans auparavant et il a le besoin de se ressourcer comme son ami Pilioko l'avait fait autrefois à Wallis. Les deux artistes partent découvrir la Russie en espérant y retrouver des traces de la famille de Nicolaï. C'est l'occasion pour Aloi de découvrir des paysages et des peuples nouveaux, il visite Léningrad redevenue Saint-Pétersbourg et Moscou, puis sur le chemin du retour vers la France, la Scandinavie, les pays d'Europe centrale, la Grèce, l'Italie et le Portugal. Les années suivantes sont

2. Finalement, quelques années après l'indépendance du pays, un nouveau Centre culturel du Vanuatu est réalisé dans la capitale Port-Vila sur un terrain près du Parlement, par l'architecte François Raulet, et l'artiste Ralph Regenvanu en est nommé directeur. Un édifice voisin, construit en matériaux traditionnels, à la manière des nakamal d'autrefois, est destiné aux réunions du Conseil national des chefs coutumiers. Ces nouveaux bâtiments viennent remplacer l'ancien Centre culturel du centre ville, initié, jadis, par Maître Desgranges et qui fut longtemps dirigé par Kirk Huffman. 


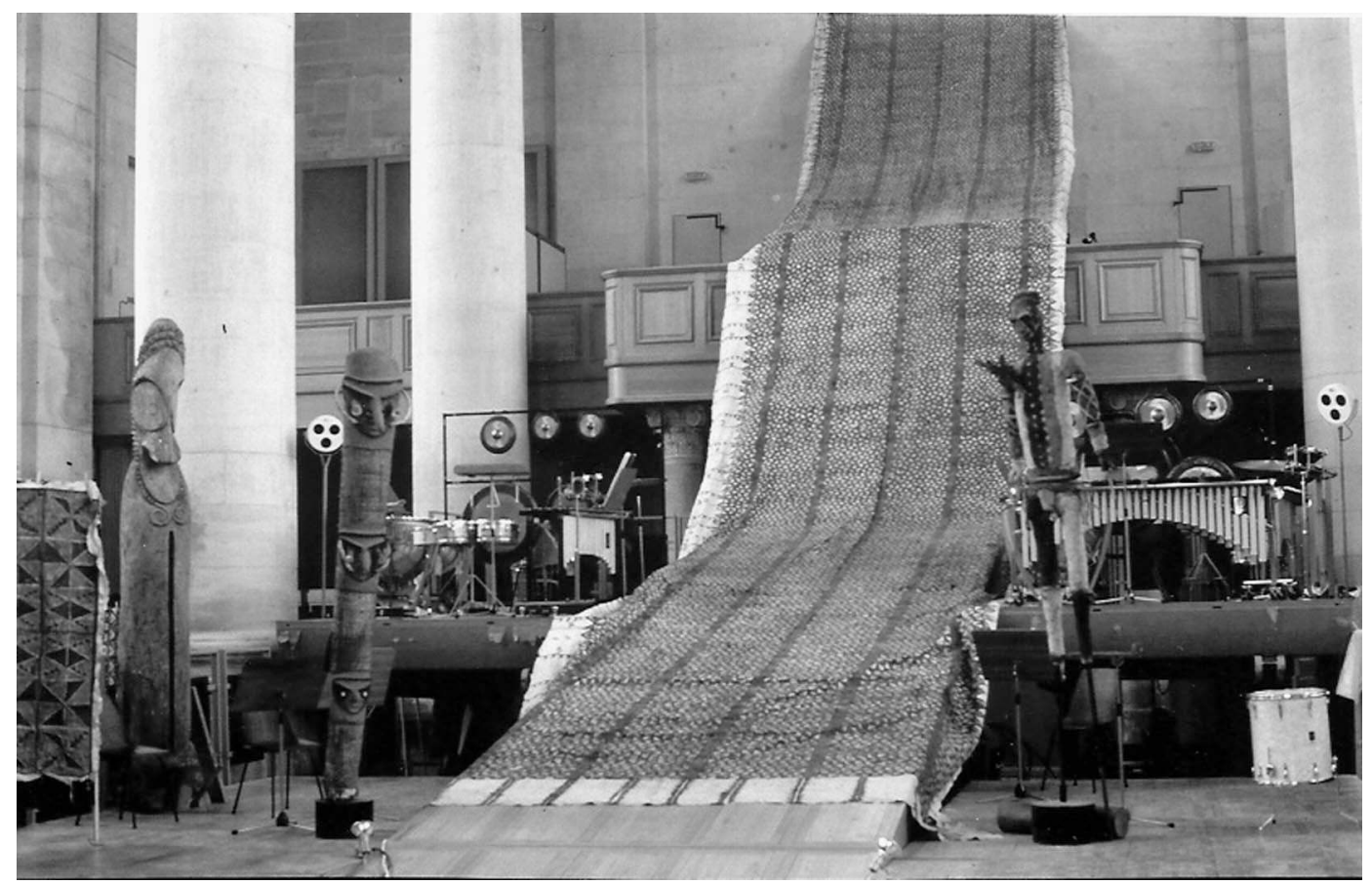

FIGURE 2. - Grand tapa de Tonga exposé au milieu d'autres œuvres d'art océaniennes sous les voûtes de la chapelle de l'Abbaye des Prémontrés (cliché Coiffier, 1978).

jalonnées d'expositions itinérantes importantes, à la Konsthall de Malmö en Suède (Bornholm, 1978), au Centre Culturel de l'Abbaye des Prémontrés à Pont-à-Mousson en France (1978). Un grand tapa de Tonga de 40 mètres de long y est présenté au beau milieu de l'abbatiale en compagnie d'un majestueux tambour d'Ambrym, d'un impressionnant rambaramb et de nombreux autres objets océaniens. Ce mélange de genres plaît beaucoup à Nicolaï qui commente ainsi la scène :

« Vous imaginez, des totems dans une abbaye construite au moment où les premiers missionnaires partaient pour évangéliser le Pacifique!» (Mwà véé, $2004: 27)$

Cette vision souvent critique de l'évangélisation des peuples du Pacifique par les missions chrétiennes n'empêche pas les deux artistes de réaliser de très nombreuses œuvres destinées à diverses institutions religieuses en Océanie (Michoutouchkine, 1971 : 36).

Un événement vient bouleverser le quotidien des deux artistes, lorsqu'en mars 1979, ils sont invités, sur proposition des académiciens Alekseï Okladnikov et de Yuriy Bromley, par l'Académie des Sciences de l'URss à l'occasion du XIV ${ }^{\mathrm{e}}$ Congrès des sciences du Pacifique qui se tient à Moscou et à Khabarovsk. À cette époque, l'aca- démie des Sciences s'intéresse aux voyages de l'explorateur et ethnologue Nikolaï Nikolayevitch Mikloukho-Maklay, qui a sillonné le Pacifique dans la deuxième moitié du $\mathrm{XIX}^{\mathrm{e}}$ siècle en réalisant de nombreux dessins et croquis d'une très grande qualité. De plus, ce dernier est revenu en Russie avec un Papou. Le duo russo-wallisien représente donc la parfaite image pour évoquer le savant explorateur et son compagnon, d'autant plus qu'il y a une homonymie au niveau des initiales N.N.M. avec celles du peintre Nicolaï Nicolayevitch Michoutouchkine (Mwà véé, 2002 : 57). De 1979 à 1986, les deux artistes vont présenter leurs collections avec leurs œuvres personnelles dans diverses villes d'Union soviétique où ils sont accueillis avec enthousiasme et ferveur par des milliers de visiteurs; de Moscou à Khabarovsk comme à Leningrad, Novosibirsk en Russie, Tbilissi en Géorgie, Sardarapat et Erevan en Arménie, Frounzé en Kirghizie, Samarcand et Tachkent en Ouzbekistan. Durant ces années, l'exposition itinérante parcourt plus de $100000 \mathrm{~km}$ et reçoit plus d'un million de visiteurs. De nombreux articles dans les langues les plus diverses sont publiés, relayés par des émissions de radio et de télévision (Gaudron, 1983). L'Institut d'ethnographie de l'académie des sciences de l'urss édite en russe (Bromley, 1985) un petit catalogue broché de leur collection avec 
le concours du Docteur Lyudmila Ivanova, spécialiste de l'art océanien. Un catalogue relié, illustré en noir et en couleurs, est ensuite traduit en français et en anglais (Bromley, 1989). Les sept cents objets et œuvres y sont présentés selon un classement fonctionnel ${ }^{3}$.

\section{Le temps de la reconnaissance}

Après des expositions à Varsovie, Townsville pour le $7^{\mathrm{e}}$ Festival des Arts du Pacifique, au Japon, à Taiwan, en Thaïlande, en Malaisie, à Singapour et en Indonésie, la collection rentre enfin à Vanuatu en 1993. Ces expositions ont été le plus souvent associées à des spectacles chorégraphiques et musicaux. On imagine difficilement la somme de travail et d'énergie déployée par les deux hommes, qui ne sont pas des professionnels de la muséographie, pour contacter les autorités douanières et culturelles, mettre en caisse, déménager, assurer et déballer toutes les pièces de leurs collections itinérantes durant ces dernières années. Une grande fête est alors organisée à Esnaar pour célébrer ce retour, avec à cette occasion un défilé de mode et de nombreuses animations. De 1993 à 2000, c'est encore plusieurs dizaines d'expositions en Europe, en Asie et Océanie, qui sont organisées dans des musées, universités, centres culturels français et divers centres de vacances Club Méditerranée. La liste en serait trop longue ( $c f$. TeissierLandgraf, 1995 : 101-102). Les deux artistes ont recueilli de nombreuses récompenses honorifiques pour leurs œuvres. Aloi Pilioko a reçu diverses médailles d'or : en 1972, au Salon des Arts et Techniques pour la qualité de ses tapisseries, en 1973, au salon de mai de Nouméa pour l'ensemble de son œuvre, en 1980, à l'Université du Pacifique-Sud à Fidji et en 1982, à l'Académie italienne Leonardo da Vinci. En 1980, il est nommé chevalier de l'Ordre du Mérite national et chevalier de l'Ordre des Arts et Lettres à Paris, en 1989. Le 30 juillet 2000, le ministre Barak Sope lui décerne, au nom du président de la république du Vanuatu, une médaille attestant du bien-fondé de ses activités culturelles pour le pays. En 2001, le maire de Port-Vila lui remet la médaille honorifique de la capitale et en 2002, il devient, ainsi que son ami Nicolaï, officier des Arts et Lettres. Leur œuvre a été saluée par les plus hautes autorités de l'état de Vanuatu, comme le Premier ministre Walter Lini et le président A. G. Sokomanu. La poste de
Polynésie française célèbre les deux artistes par l'édition de deux timbres, en 1971 et en 1972. De son côté, la poste de Wallis et Futuna honorera les couleurs chatoyantes des tapisseries d'Aloi Pilioko par un timbre édité en 1982. Un travail universitaire d'histoire de l'art, intitulé : Aloi Pilioko, un singulier artiste entre coutume et contemporanéité, soutenu en septembre 2005 à l'université de Lyon, vient d'être réalisé sur l'œuvre de Pilioko par madame Hélène de Mongolfier.

Tous deux ont aidé de façon discrète, mais efficace, de nombreux jeunes artistes du Pacifique au début de leur carrière. Nicolaï excelle en communication car il adore mettre les gens en relation. Aux mois de mai 1984 et 1985, la Fondation Michoutouchkine-Pilioko sur le site d'Esnaar, est choisie par l'Institute of Pacific Studies et la South Pacific Creative Arts Society pour l'organisation de deux ateliers de formation destinés à encourager, stimuler et promouvoir de jeunes artistes et artisans en provenance de divers archipels du Pacifique, comme Pierre Chanel Simutoga (1992) de Wallis. Les artistes travaillent séparément ou en ateliers collectifs, mais se rencontrent aussi fréquemment pour confronter leur point de vue sur l'art et notamment sur la manière de le faire participer à la vie de tous les jours (Gaudron, 1983 : 45-46, Hereniko, 1986). Au cours de ces ateliers, financés par la Fondation, Michoutouchkine et Pilioko s'efforcent de faire prendre conscience à cette nouvelle génération d'artistes de l'influence exercée par les créations de leurs ancêtres sur l'art contemporain international. Ils souhaitent créer une nouvelle dynamique en s'appuyant sur ces paroles de Michael Somare, Premier ministre de Papouasie Nouvelle-Guinée :

« Nous rejetons la façon dont on nous a considérés comme des objets passifs de recherche et d'études pour les experts. Nous voulons partager notre culture et notre art avec des gens qui apprécient les Beaux-Arts du Pacifique. » (Somare, 1983 : 97)

Nicolaï a toujours défendu l'idée d'une continuité historique et factuelle entre l'art dit traditionnel et l'art contemporain. Ces ateliers eurent un grand succès et une influence certaine sur le développement de l'art contemporain dans la région (Regenvanu, 1996 : 322-323), la création de l'association Nawita leur doit certainement beaucoup (Mwà véé, $2002: 56$ ). Ils seront ensuite décentralisés sur Fidji, Rarotonga et Tonga. Il existe ainsi maintenant dans tous les archipels du

3. Michoutouchkine vient de traduire du russe en français des extraits de l'histoire de la corvette Diana et du capitaine Vasilii Golovnin, premier navigateur européen à visiter, en 1809, l'île de Tanna après le capitaine James Cook. Cette traduction a été publiée récemment dans le journal La Presse à Vanuatu. 
Pacifique une relève de jeunes artistes qui ne demandent qu'à s'exprimer, comme Rébecca Hoatau, Raphaël Kaikilekofe et Soane Papita Takaniua de Wallis qui se sont déjà fait remarquer dans divers salons.

En ce qui concerne la création contemporaine, Michoutouchkine critique tout à la fois la main mise des religieux et celle de certains chefs coutumiers qui empêchent les artistes océaniens de s'exprimer librement en ne cherchant pas à comprendre leur démarche créatrice. Il dénonce le fait que les peintres du Pacifique soient rapidement étouffés par leur famille et leur entourage dès qu'ils commencent à vivre de leur art (Mwà véé, 2002: 52). Sa conception de la muséographie va à l'encontre de celle de la majorité des spécialistes actuels et l'anti-musée d'Esnaar en est un bon exemple. Ainsi, il défend toujours la mise à portée du public, hors vitrine, des objets pour que s'établisse un dialogue véritable entre ces derniers et l'observateur, au sein de la nature. Il a d'ailleurs toujours privilégié l'exposition de ses collections et de ses ouvres picturales dans de petites localités comme à Santo à Vanuatu, Bourail en NouvelleCalédonie, Atuona aux îles Marquises, Honiara aux Salomon... pour présenter l'art océanien aux Océaniens eux-mêmes. Sa conception de la relation à l'objet est cependant tout à fait actuelle lorsqu'il dit :

« Les explications qui vont avec l'objet sont certes importantes, mais moins que la rencontre avec l'objet lui-même, moins que l'émotion qu'il crée. " (Mwà véé, $2002: 52)$

Il est certain que l'émotion ressentie par Nicolaï est très différente de celle d'Aloi qui a vécu avec des objets similaires depuis sa plus tendre enfance. Le mysticisme avoué de Nicolaï l'a toujours fait se méfier des scientifiques et il admet que son intérêt pour les sciences occultes et les expériences supranaturelles l'a spontanément poussé vers les pays d'Orient (Teissier-Landgraf, 1995 : 79).

Aloi Pilioko tire son inspiration de sa propre culture wallisienne tout en utilisant des moyens d'expression nouveaux comme la tapisserie. Ses œuvres réalisées à l'aide de fils de laine de couleurs vives sont d'ailleurs les plus appréciées. Elles sont achetées tant par des collectionneurs $\mathrm{du}$ Pacifique que d'Europe et d'Asie. Aloi est resté très humble devant le succès :

«[Il] a su maintenir dans son œuvre la qualité et l'originalité qui caractérisent le véritable artiste. Il n'existe pas dans sa production deux tapisseries qui soient semblables. » (Tausie, 1981 : 104)
Le succès de Pilioko est dû en grande partie au soutien sans faille que lui a manifesté depuis plusieurs dizaines d'années son ami Nicolaï. Ce dernier a su créer à Esnaar une atmosphère idéale pour permettre à l'artiste d'épanouir son esprit créatif, de travailler et de s'exprimer dans les meilleures conditions.

Aujourd'hui encore, les deux artistes continuent de créer de nouvelles collections de mode océanienne avec des tissus peints par eux-mêmes, chemises, robes, shorts, paréos, que l'on peut admirer dans leur boutique de la rue principale de Port-Vila. Les acheteurs de ces pièces uniques et originales deviennent alors des tableaux ambulants, hauts en couleurs, porteurs d'un message de joie. Depuis 1985, ils puisent une énergie nouvelle en organisant dans différentes villes d'Océanie des présentations de mode qui ont toujours un grand succès (Gaudron, 1983 : 35-36). Nicolaï déclare ainsi :

« J'ai voulu peindre toutes les couleurs d'air qui rappellent les paysages, les cérémonies, afin de les faire revivre et bouger par les mannequins de mes défilés de mode. » (Teissier-Landgraf, 1995 : 89)

Certains ont pu déplorer que ces artistes confirmés et reconnus se contentent de reproduire (même si chacune des pièces est unique) sur de modestes tissus de cotonnade, et de manière un peu trop commerciale, les thèmes et les motifs qui ont fait leur succès par le passé. C'est ignorer le problème qui se pose à tout artiste. Que faire pour durer en vivant de son art ? Cette activité leur permet de se renouveler et de continuer à vivre à leur manière dans leur propriété d'Esnaar, dont les bâtiments ont été plusieurs fois détruits par des cyclones et reconstruits. Le jardin où se mélangent végétation tropicale, aux fleurs colorées et odoriférantes, et divers spécimens de l'art monumental océanien, est entretenu par Aloi qui y nourrit chaque matin ses coqs, ses chiens et ses chats. Ce sont ces derniers qui stimulent son imagination créatrice. La salle à manger de plein air, face à la mer, a vu passer de très nombreuses personnalités des arts, des lettres, des sciences, de la mode, de la politique et de la diplomatie. Leurs très nombreux ami(e)s gardent tous un souvenir ému des sympathiques dîners aux chandelles et des mets originaux, toujours préparés avec art, par leurs hôtes.

Aloi Pilioko est l'un des premiers artistes polynésiens dont la réputation a dépassé son île pour atteindre tout le Pacifique, l'Europe et l'Asie (Michoutouchkine, 1982:27). Si sa timidité et sa retenue naturelle recueillent l'assentiment général, la personnalité exubérante, voir hâbleuse de Nicolaï irrite et énerve certains, ce qui lui vaut 


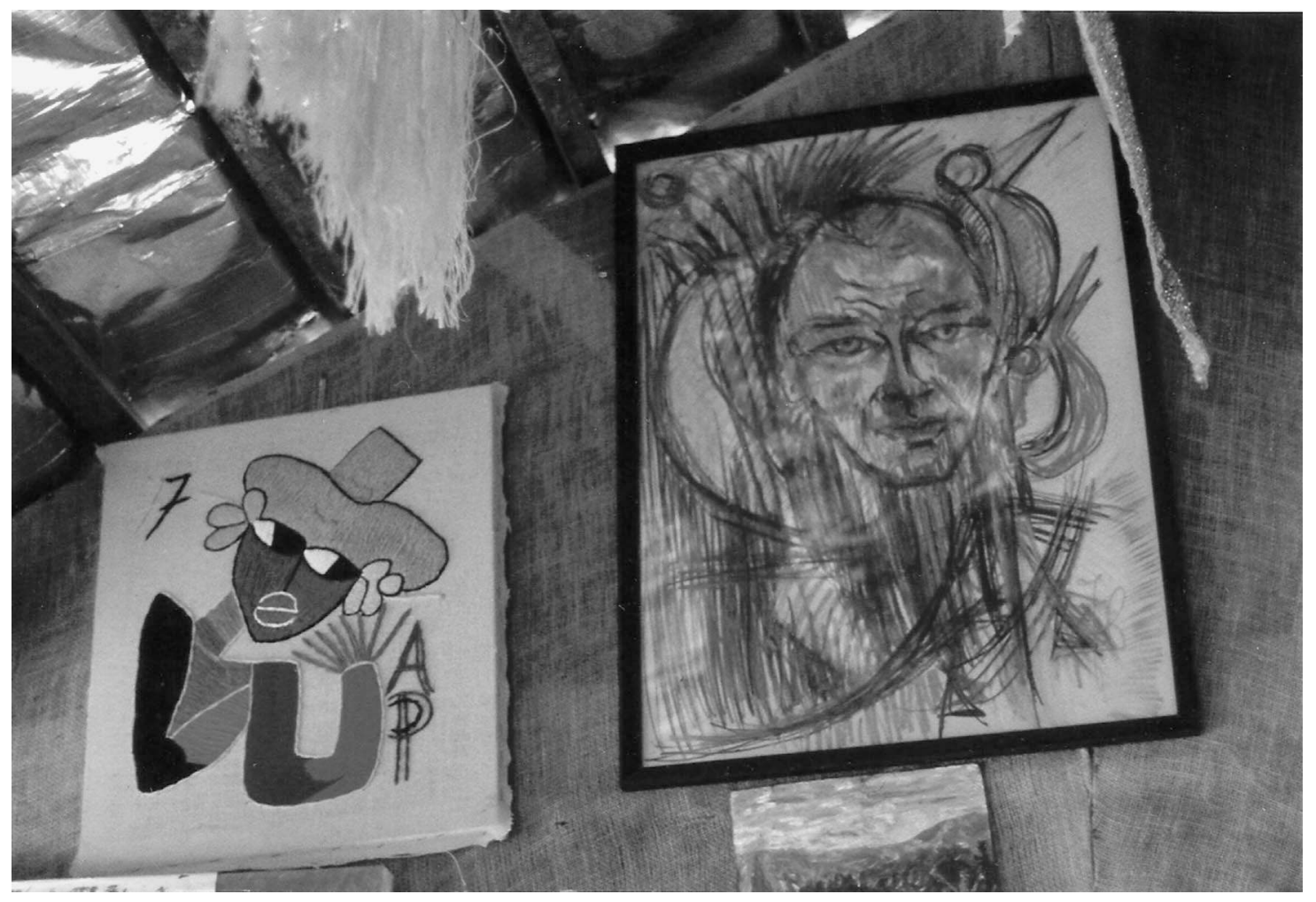

Figure 3. - Auto-portraits d'Aloi Pilioko (à gauche) et de Nicolaï Michoutouchkine (à droite) accrochés à un mur de leur ancienne demeure d'Esnaar (cliché Coiffier, 1979)

leur inimitié dont il n'a cure. Il fait sienne cette parole de Jean Cocteau "Ce que le public te reproche, cultive le, c'est toi!» (TeissierLandgraf, 1995 : 95). Quelqu'un a écrit à son propos : il dérange parce que son génie est envahissant. Michoutouchkine est un univers à lui tout seul car il transgresse les modes, les régimes et les barrières linguistiques. Jacques Gaudron a décrit les deux hommes ainsi :

« de tempérament opposé, "l'esprit dominateur de l'un n'a d'égal (que) la docilité de l'autre" les deux peintres d'une affabilité qu'il est actuellement si difficile de trouver dans ce monde, laissent percevoir un sens aigu des autres. » (Gaudron, 1983 : 21)

Nicolaï est un peintre doublé d'un collectionneur «militant», suivant la formule de F. Fay (Michoutouchkine, 1971 : 33). C'est un provocateur sur le plan artistique et un sauveteur d'objets d'ethnographie en perdition (idem : 44). Il ne collectionne pas par souci d'accumulation, mais pour montrer les objets, pour jeter des ponts culturels entre les communautés mélanésiennes et polynésiennes séparées par les aléas de l'histoire et de la colonisation. Il regrette amèrement que des milliers d'objets restent enfouis dans des réserves des musées les plus connus du monde et soient ainsi soustraits aux regards des peuples qui en ont le plus besoin pour retrouver leur identité ou pour la conserver (Michoutouchkine \& Pilioko Foundation, 1982: 38). Jean Gabus (Egami, 1980:7) écrit à son sujet que :

« [le peintre] sait façonner les objets à la mesure de sa passion, les humanise. Hors de leur milieu d'origine, les objets ne sont le plus souvent que des œuvres mortes, en particulier dans les musées. ».

Si Michoutouchkine a été parfois vilipendé par certains acteurs officiels de la Culture qui lui reprochent de réaliser ses expositions trop rapidement, sans préparation suffisante et sans précaution pour les objets, il faut reconnaître, qu'avec son ami Pilioko, ils ont accompli, avec des moyens financiers réduits, ce qu'aucune institution culturelle publique n'avait réussi avant eux. La formation commerciale de Nicolaï lui a permis de comprendre bien avant d'autres qu'il était possible d'organiser des expositions rentables en promouvant les arts du Pacifique. Son principe a toujours été que les revenus d'une exposition, avec la vente de ses œuvres et de celles de Pilioko, devaient financer les prochaines expéditions et les achats d'objets destinés à des expositions futures (Egami, 1980 : 118). Leur désir est de faire rayonner la connaissance des arts du 


\section{May 1606 - May 2006

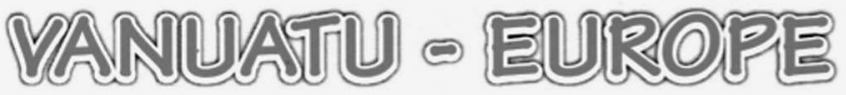 400th Anniwersary}
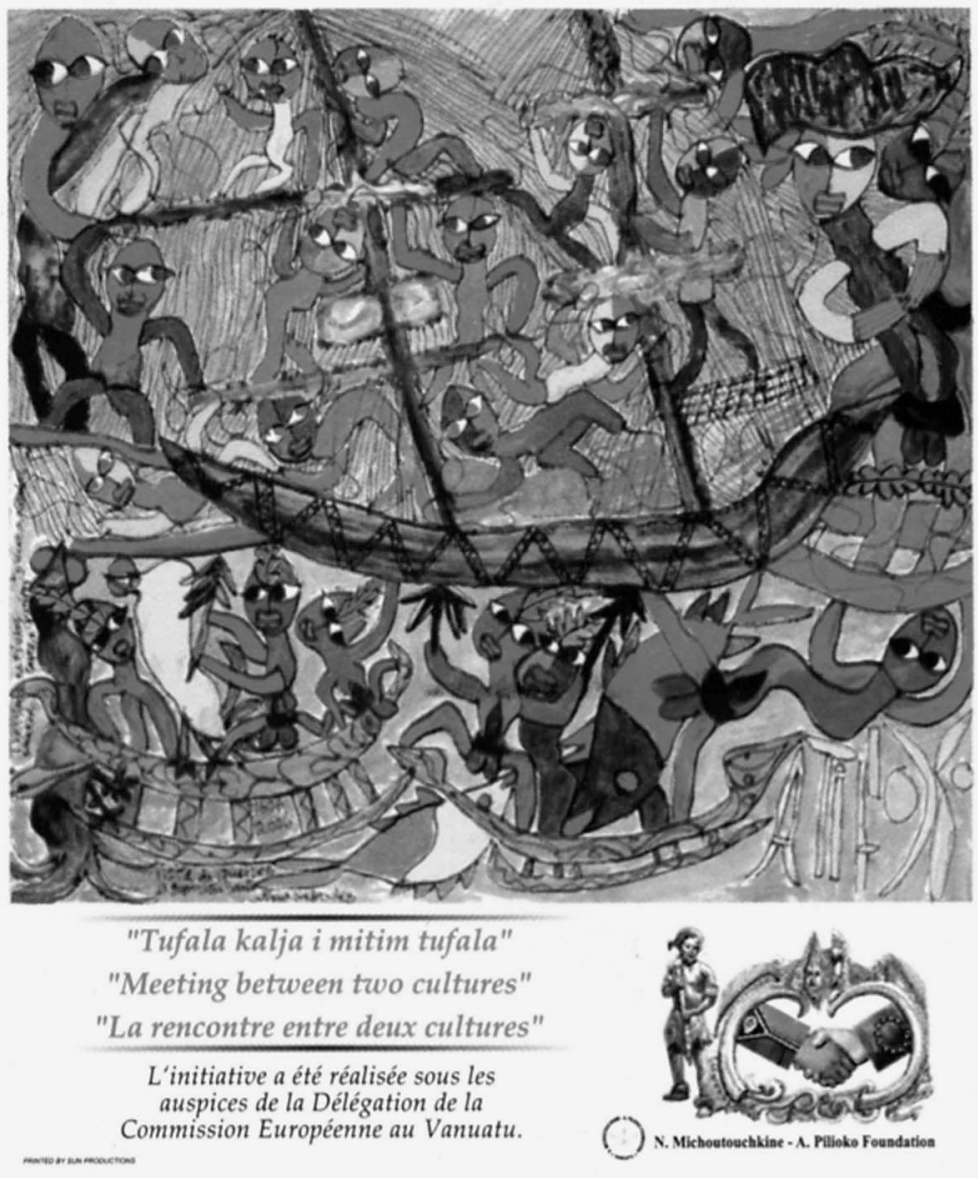

FIGURE 4. - Dernière œuvre réalisée en 2006 par Aloi Pilioko. Elle représente les premiers contacts entre les Européens et les peuples du Vanuatu (C Fondation N. Mouchoutouchkine - A. Pilioko)

Pacifique à partir de leurs lieux de création pour le bénéfice des intéressés eux-mêmes, les peuples océaniens. Certains détracteurs leurs reprochent d'avoir revendu des objets anciens qu'ils avaient acquis précédemment dans les villages. Mais ce sont eux qui ont encouragé Paul Gardissat à publier en 2004 une anthologie de la littérature orale du Vanuatu constituée de contes et de légendes enregistrés à travers l'archipel lorsque l'auteur travaillait pour Radio-Port-Vila. Il est difficile de répertorier toutes les activités des deux artistes tant celles-ci ont été nombreuses et variées depuis plus de quarante-cinq ans. Cette profusion se retrouve dans les catalogues rétrospectifs où transparaît la personnalité passionnée et impatiente de Nicolaï, avec ce désir boulimique de montrer pêle-mêle tous ses souvenirs : photos, coupures de journaux, croquis, etc. (Fondation Michoutouchkine-Pilioko, 1982, Gaudron, 1983).

\section{Conclusion}

Aujourd'hui, l'informatique, avec le réseau Internet, permet de visionner à distance les collections des musées pourvu que chaque objet avec sa fiche ait été numérisé dans une banque de données. Ce sera la grande nouveauté du futur musée du quai Branly de permettre à des millions d'internautes de consulter à distance les centaines de milliers d'objets, dont plus de trente mille d'Océanie, de ses collections. Cette nouvelle technique devrait contribuer à exaucer le rêve 
des deux artistes de Port-Vila : la promotion des cultures insulaires du Pacifique. Cependant, la vision virtuelle d'un objet ne remplacera certainement pas sa perception de visu pour laquelle ils ont dépensé tant d'énergie durant plus de quarante ans. Aloi Pilioko et Nicolaï Michoutouchkine se revendiquent les ambassadeurs de la culture océanienne à travers le monde, raison pour laquelle ils ont délibérément choisi de rester en Océanie, dans une petite ville comme Port-Vila, plutôt que de s'installer dans le confort discutable (à leurs yeux) d'une métropole comme Sydney, Auckland ou Honolulu. Le terrain d'Esnaar vient d'être classé, en 2005, site historique national par le gouvernement du Vanuatu. Infatigables, les deux artistes préparent actuellement, en 2006, une nouvelle exposition permanente avec environ 200 pièces de leur collection, associées aux peintures qui avaient servis à l'acquisition du terrain d'Esnaar et qui viennent d'être rachetées à la famille de maître Desgranges (supra). Toutes ces œuvres devraient être exposées prochainement dans un nouveau musée de l'Asie et du Pacifique à Nusa Dua dans l'île de Bali. Aloi Pilioko et Nicolaï Michoutouchkine représentent l'exemple rare, voire unique, d'une symbiose réussie entre deux êtres et deux sensibilités très différentes, l'une polynésienne, l'autre slave, dont l'œuvre commune et pourtant distincte reflète l'expression de leur longue amitié et l'amour de tout ce qui concerne les peuples et les cultures du Pacifique. Ils auront transmis durant près d'un demi-siècle une vision très particulière du Pacifique.

\section{BIBLIOGRAPHIE}

Bornholm Ann, 1978. Söderhavskonst, Malmö (Suède), Konsthall.

Bromley Yuriy, 1985. Catalogue de l'exposition Ethnographie et Art de l'Océanie de la Fondation $N$. Michoutouchkine-A. Pilioko (version en langue russe), Moscou, ministère de la Culture et académie des sciences de l'urss.

—, 1989. Catalogue de l'exposition Ethnographie et Art de l'Océanie de la Fondation N. Michoutouchkine-A. Pilioko, traduction française d'E. Bronina, Moscou, ministère de la Culture et académie des Sciences de l'urss.

COIFFIER Christian, 1988. Traditional Architecture in Vanuatu, Port-Vila, Suva, Institute of Pacific Studies and the Vanuatu Extension centre of the University of the South Pacific.

_, 2000. Sculpture de Malo, Grande sculpture masculine (Trrou Körrou), in Jacques Kerchache (éd.) Sculptures, Afrique, Asie, Océanie, Amériques, Paris, RMN/musée du quai Branly, pp. 274-278.
Crocombe Ron G., 1973. The New South Pacific, Auckland, Sydney, London, Reed Education.

Egami Namio, 1980. Arts et Traditions Populaires de l'Océanie, Nagoya (Japon), The Little World of Man.

GaIllot Marcel, 1963. Nicolaï Michoutouchkine, peintre à Futuna, Journal de la Société des Océanistes 19, pp. 211-214.

Gardissat Paul, 2004. Nabanga, une anthologie illustrée de la littérature orale du Vanuatu, Port-Vila, Centre culturel du Vanuatu/Conseil culturel national du Vanuatu.

GAUDRON Jacques 1983. Vingt-cinq ans de référence, Port-Vila, Michoutouchkine \& Pilioko Foundation.

Hereniko Patricia (ed.) 1986. Pacific Artists, Suva, Fiji, University of the South Pacific.

Michoutouchkine Nicolaï, 1970. Catalogue de la collection d'objets d'arts du Pacifique, Port-Vila, Imprimerie Hébridaise, Arts du Pacifique 2.

_, 1971. Hommage à Nicolas Mikloukho-Maklay, Tahiti, Maeva Beach Hotel, Arts du Pacifique 3.

-, 1975. Vers un musée vivant, Bulletin du Pacifique Sud 25, 3, pp. 52-55.

Michoutouchkine \& Pilioko Foundation 1982. Océanic Art Océanien, rétrospective de cinq années d'expositions (1978-1982), Port-Vila (Vanuatu).

Montgolfier (de) Hélène, 2005. Aloi Pilioko, un singulier artiste entre coutume et contemporanéité, mémoire de Master d'histoire de l'art, Lyon, université Lumière - Lyon II.

Mwà véé 37, 2002. Nicolaï Michoutouchkine et Aloi Pilioko et Ambiance artistes avec Nawita, Nouméa, ADCK/Centre culturel Tjibaou, pp. 49-58.

— 45, 2004. Nicolaï Michoutouchkine et Aloi Pilioko, ambassadeurs de l'Océanie à travers le monde, Nouméa, ADCK/Centre culturel Tjibaou, pp. 53-61.

Pilioko Aloi, 1980. Aloi Pilioko, Artist of the Pacific, Artiste du Pacifique, Vila, Vanuatu, South Pacific Social Science Association/Institute of Pacific Studies/University of the South Pacific.

Regenvanu Ralph, 1996. L'art contemporain au Vanuatu, in J. Bonnemaison, K. Huffman, C. Kaufmann et D. Tryon, Vanuatu Océanie, Arts des îles de cendre et de corail, Paris, RMN/ORSTOM, pp. 322-329.

SImutoga Pierre-Chanel, 1992. Technologie traditionnelle à Wallis. Essai de sauvegarde de la mémoire collective des charpentiers wallisiens (tufuga) du district de Hihifo, Paris, Publications de la Société des Océanistes 44.

Somare Michael, 1983. Perspectives du Pacifique : problèmes et avenir des peuples insulaires, Port-Vila (Vanuatu), Centre de l'Université du Pacifique Sud/ Association des sciences sociales du Pacifique Sud, pp. 87-100.

Teissier-Landgraf Marie-Claude, 1995. Le Russe de Belfort, 37 ans de voyages du peintre Nicolaï Michoutouchkine en Océanie, Suva (Fiji) Institute of Pacific Studies, The University of the South Pacific. 
Tausie Vilsoni, 1981. Art du Pacifique, La question de l'art dans un Pacifique en pleine évolution, traduit de l'anglais par Éric Le Moal, Suva, Fiji, Institut des Études sur le Pacifique/Centre de Port Vila au Vanuatu/université du Pacifique Sud.
Remerciements - L'auteur remercie Tatiana Benfoughal qui a vérifié et corrigé les noms russes. 